\title{
Successful Application of Extracorporeal Membrane Oxygenation for a Patient with Clinical Amniotic Fluid Embolism
}

\author{
Hye Seon Kang, M.D. ${ }^{1}$, Hwa Young Lee, M.D. ${ }^{2}$, Hea Yon Lee, M.D. ${ }^{2}$, and Seok Chan Kim, M.D., Ph.D. ${ }^{2}$ \\ 'Division of Pulmonary, Allergy and Critical Care Medicine, Department of Internal Medicine, Incheon St. Mary's Hospital, College of Medicine, The Catholic University of Korea, \\ Incheon; 'Division of Pulmonary, Allergy and Critical Care Medicine, Department of Internal Medicine, Seoul St. Mary's Hospital, College of Medicine, The Catholic University of Korea, \\ Seoul, Korea
}

Amniotic fluid embolism (AFE) is a rare but potentially fatal complication that occurs acutely during pregnancy or within $12 \mathrm{~h}$ of delivery. The management of AFE focuses initially on supportive measures for cardiopulmonary stabilization. Extracorporeal membrane oxygenation should be considered in patients who are unresponsive to medical treatment in order to prevent additional hypoxia and subsequent organ failure. We present a 41-year-old woman with clinical AFE who developed acute respiratory distress syndrome and was treated successfully with extracorporeal membrane oxygenation.

Key Words: adult; amniotic fluid; embolism; extracorporeal membrane oxygenation; respiratory distress syndrome.

Amniotic fluid embolism (AFE) is a potentially fatal complication that occurs acutely during pregnancy or within $12 \mathrm{~h}$ of delivery.[1,2] AFE is rare, with an overall incidence in the range of 1 in 8,000 to 1 in 80,000 deliveries.[3] The mortality rate associated with AFE is high, approaching $60 \%$, although the prognosis has improved significantly with early recognition and prompt supportive measures.[2,3] The treatment of AFE is based on supportive treatment for cardiopulmonary stabilization. However, there is no treatment modality in cases unresponsive to medical therapy.[4] We herein present a case involving a patient with clinical AFE, whose condition progressed to refractory acute respiratory distress syndrome, for which veno-venous (VV) extracorporeal membrane oxygenation (ECMO) was required. She was treated successfully with ECMO.

\section{Case Report}

A 41-year-old woman was admitted to the emergency department with gross hematuria after dilation and curettage (D\&C). She was at 19 weeks of gestation (according to her last menstrual period) with an uncomplicated pregnancy, and she had an

Received on July 31, 2015 Revised on September 10, 2015 Accepted on September 10, 2015

Correspondence to: Seok Chan Kim, Division of Pulmonary, Allergy and Critical Care Medicine, Department of Internal Medicine, Seoul St. Mary's Hospital, College of Medicine, The Catholic University of Korea, 222, Banpo-daero Seocho-gu, Seoul 06591, Korea

Tel: +82-2-2258-6062, Fax: +82-2-599-3589

E-mail: cmcksc@gmail.com

*No potential conflict of interest relevant to this article was reported. obstetric history of gravida 3 , para 1 , abortion 2. Computed tomography cystography showed intraperitoneal bladder rupture with suspected $3.8-6.3-\mathrm{cm}$ hematoma near the left base of the bladder. Fluid and air bubbles were noted in the uterus. Her vital signs were a heart rate of 72 beats/min and blood pressure of 83/54 mmHg. The level of hemoglobin was $14.8 \mathrm{~g} / \mathrm{dL}$. Three hours after D\&C performed at local obstetric clinic, she underwent laparoscopic bladder injury repair 

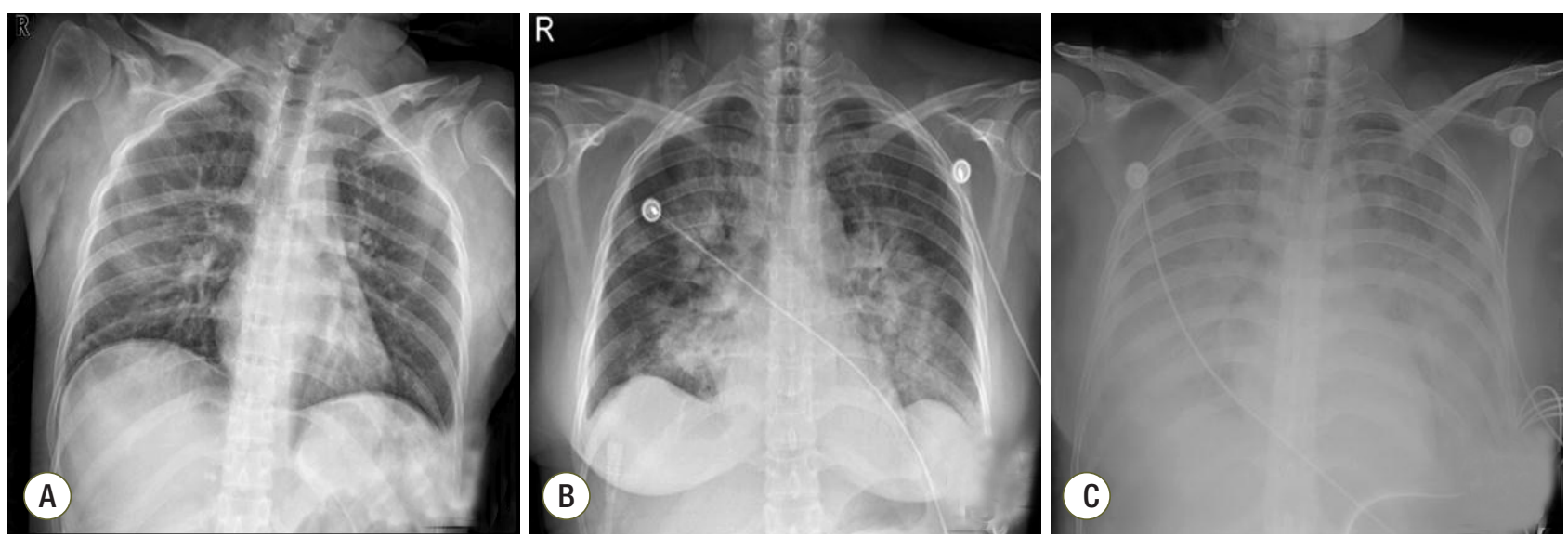

Fig. 1. Chest radiographs at (A) $3 \mathrm{~h},(\mathrm{~B}) 12 \mathrm{~h}$, and (C) $20 \mathrm{~h}$ after dilatation and curettage (D\&C).
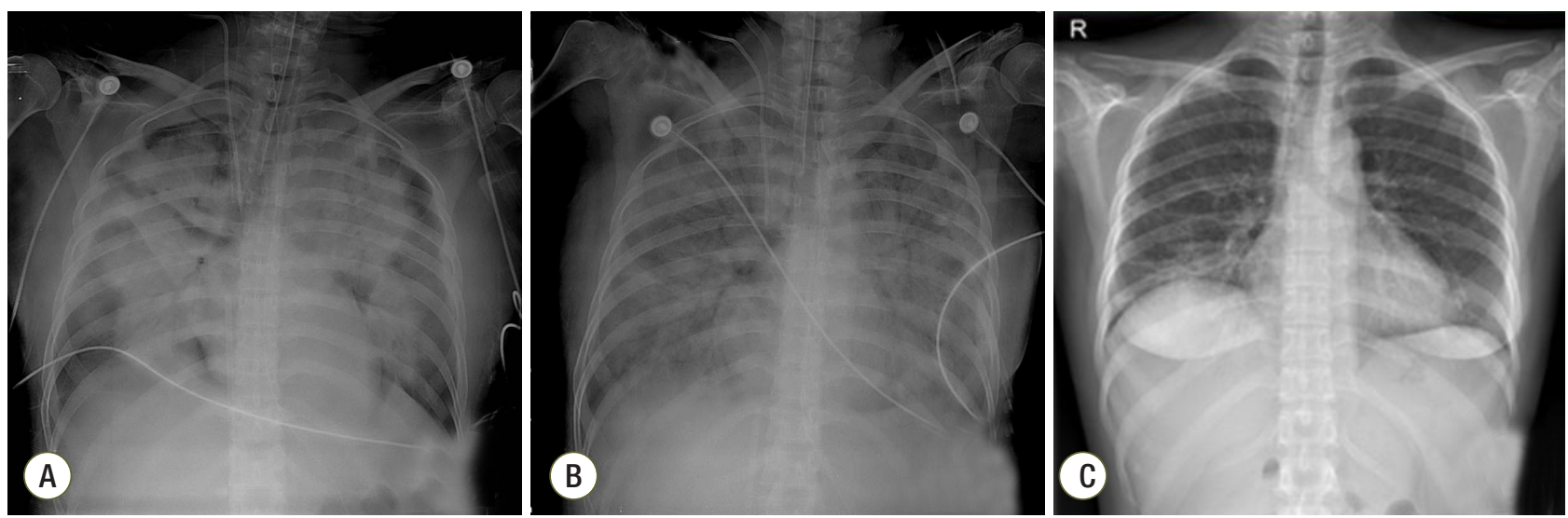

Fig. 2. Chest radiographs $(A)$ on day 1 of extracorporeal membrane oxygenation (ECMO), $(B)$ improved consolidations in both lungs on day 11 of ECMO, and (C) in the out-patient clinic seven days after discharge.

and primary closure of the uterine wall with foreign body removal. She did not receive a blood transfusion during the operation. The total operative time was $2 \mathrm{~h}$, and there was no volume overload during the operation. A sudden episode of dyspnea and chest tightness occurred $2 \mathrm{~h}$ postoperatively. Chest radiography showed newly developed pulmonary edema (Fig. 1). There were no obvious petechial rashes or blood stained sputum. The white blood cell count was $17,510 / \mathrm{mm}^{3}$ with $76.6 \%$ segmented neutrophils; the Creactive protein level was $0.48 \mathrm{mg} / \mathrm{dL}$ (reference range, $<5$ $\mathrm{mg} / \mathrm{dL}$ ); troponin-I and creatine kinase-MB was $0.526 \mathrm{ng} /$ $\mathrm{mL}$ and $3.29 \mathrm{ng} / \mathrm{mL}$, respectively; procalcitonin was 0.351 $\mathrm{ng} / \mathrm{mL}$. Empirical broad-spectrum antibiotics were initiated for presumed hospital acquired pneumonia. An echocardiogram revealed normal left ventricular systolic function with a collapsed inferior vena cava indicating hypovolemia. The blood coagulation panel was consistent with disseminated intravascular coagulation (DIC), including a fibrinogen level of $361 \mathrm{mg} / \mathrm{dL}$, antithrombin III level of $71 \mathrm{mg} / \mathrm{mL}$, Ddimer level of $6.4 \mu \mathrm{g} / \mathrm{mL}$, fibrin degradation products level of $17.66 \mu \mathrm{g} / \mathrm{mL}$, prothrombin time of $12.3 \mathrm{~s}$, partial thromboplastin time of $29.5 \mathrm{~s}$, international normalized ratio of 1.03 , and platelet count of $106,000 / \mu \mathrm{L}$. Two sets of blood cultures obtained before antimicrobial therapy demonstrated negative. AFE was highly suspected because of the clinical findings of acute hypoxia, and DIC within $12 \mathrm{~h}$ of D\&C, which could not be explained by other causes such as acute heart failure, transfusion-related acute lung injury, sepsis, or pulmonary thromboembolism. She developed acute respiratory failure requiring intubation and mechanical ventilation. Hypoxemia worsened despite advanced mechanical ventilation with increasing positive end-expiratory pressure (PEEP) (a peak inspiratory pressure of $20 \mathrm{cmH}_{2} \mathrm{O}$, respiratory rate of 18 breaths/min, PEEP of $18 \mathrm{mmHg}$, and fraction 


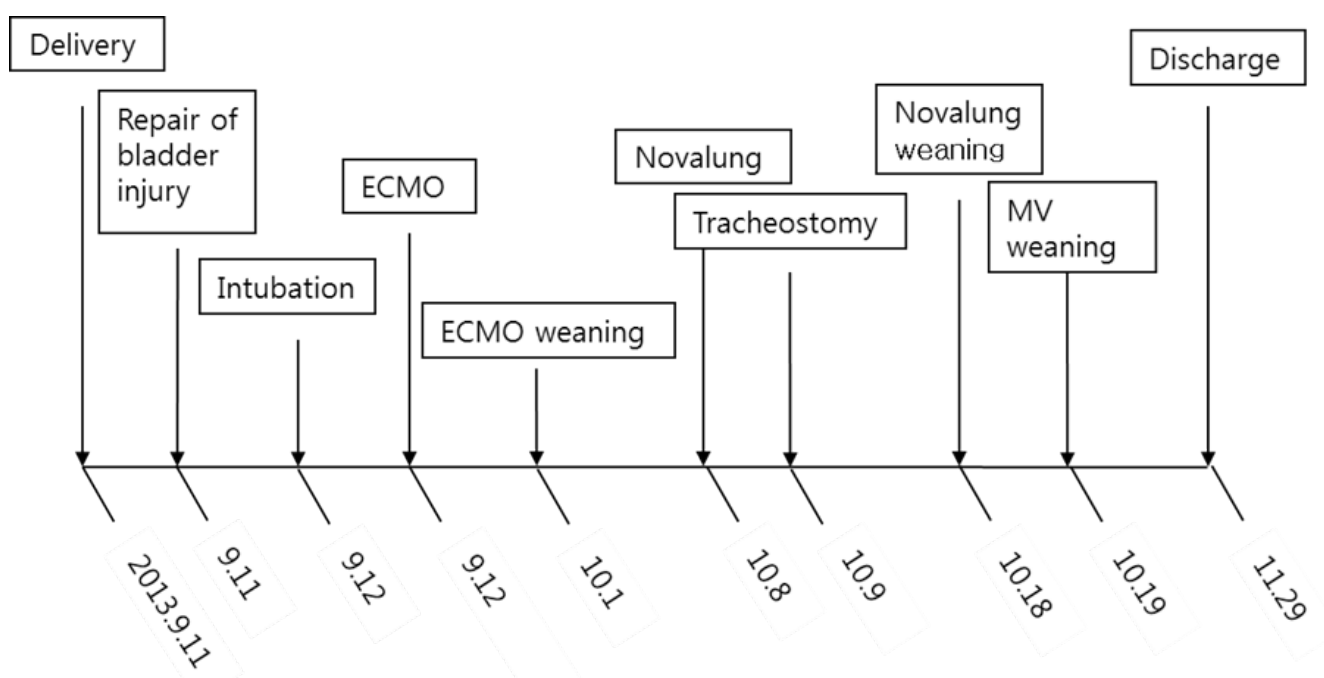

Fig. 3. Timeline of major clinical events. ECMO: extracorporeal membrane oxygenation; MV: mechanical ventilation.

of inspired oxygen $\left[\mathrm{FiO}_{2}\right]$ of 1.0 with the following arterial blood gas analysis [ABGA] results: $\mathrm{pH}, 7.40 ; \mathrm{pCO}_{2}, 30$ $\mathrm{mmHg}$; and $\mathrm{PaO}_{2}, 53 \mathrm{mmHg}$ ). Routine use of steroid for acute respiratory distress syndrome was not considered. The patient was placed on VV ECMO (Fig. 2A). We used RotaFlow $^{\circledR}$ (Maquet Inc., Hirrlingen, Germany). Under sterile conditions, a 16-French catheter and an 18-French catheter was placed in the right internal jugular vein and the right femoral vein, respectively. She was initiated with a circuit flow of $3.8 \mathrm{~L} / \mathrm{min}$ and sweep of $2.0 \mathrm{~L} / \mathrm{min}$ of oxygen $(100 \%$ fractional delivered oxygen). Concurrent ventilator settings included a peak inspiratory pressure of $25 \mathrm{cmH}_{2} \mathrm{O}$, respiratory rate of 8 breaths/min, PEEP of $15 \mathrm{mmHg}$, and $\mathrm{FiO}_{2}$ of 0.4 . Bronchoscopy on day 5 of ECMO revealed no evidence of bleeding although bronchoalveolar lavage was not performed. The patient demonstrated recovery of lung function and improved radiologic findings on day 11 of ECMO (Fig. 2B). She was weaned from VV ECMO and decannulated (cumulative ECMO duration: 456 h). However, her hypoxia and hypercarbia became worsened $\left(\mathrm{FiO}_{2}\right.$ of 0.6 with the following ABGA results: $\mathrm{pH}, 7.165 ; \mathrm{pCO}_{2}, 88.3 \mathrm{mmHg}$; and $\mathrm{PaO}_{2}, 85.2 \mathrm{mmHg}$ ) after cessation of ECMO 10 days later. Thus, she was placed on extracorporeal lung assistance (Novalung $\AA$, GmbH, Heilbronn, Germany). She was weaned from the extracorporeal lung assistance after 10 days. Her ECMO course was complicated by the development of bilateral pneumothoraces related to the artificial ventilation and multiple episodes of Acinetobacter baumannii and Can- dida spp. in her endotracheal aspiration and blood cultures, which were treated with antibiotics and antifungal agents. Her lung function continued to improve, and she was successfully weaned from mechanical ventilation (cumulative ventilator duration: 37 days). After a 59-day stay in the intensive care unit, she was transferred to the general ward for rehabilitation. At 18 days after discharge from the intensive care unit, the patient was discharged home without supplemental oxygen. On follow-up at out-patient clinic in 7 days after discharge, chest radiography showed near complete regression of the previously noted diffuse infiltrates in both lungs (Fig. 2C). Her clinical course is summarized in Fig. 3.

\section{Discussion}

AFE is a catastrophic complication that occurs acutely during pregnancy or within $12 \mathrm{~h}$ of delivery.[1,2] The overall incidence of AFE ranges from 1 in 8,000 to 1 in 80,000 deliveries.[3] The mortality rate associated with AFE is high, approaching $60 \%$, although the prognosis has improved significantly with early recognition and prompt supportive measures.[2,3] The pathogenesis of AFE is not fully understood. Traditionally, it was believed that the entry of amniotic fluid into the maternal circulation caused obstruction of pulmonary capillaries, resulting in cardiovascular collapse. Recently, a suggested possible mechanism is that the entrance of amniotic fluid into the maternal circulation 
triggers release of mediators such as histamine, bradykinin, leukotrienes, and thromboxane, leading to anaphylactic shock.[5]

AFE is diagnosed based on the patient's clinical presentation.[6] The classic triad of clinical findings is hypoxia, hypotension, and DIC. Entry criteria for national registries for suspected AFE in the United States and United Kingdom comprise four factors: 1) acute hypotension and cardiac arrest, 2) acute hypoxia, 3) coagulopathy or severe clinical hemorrhage, and 4) all of these occurring during labor, Cesarean delivery, D\&E, or the postpartum period.[3,7] However, many cases of AFE have occurred without one or more of the cardinal signs.[4] Yoneyama et al[8] reported that DIC was a major initial presenting symptom of AFE, but cardiopulmonary collapse or respiratory distress was noted in $50 \%$ of patients. Although the detection of fetal squamous cells in maternal central venous blood was considered a diagnostic method for AFE, no standard confirmatory test for diagnosis is yet available. Thus, AFE is highly suspected in the pregnant or postpartum patient who presents with acute hemorrhage with DIC, altered mental status, hypotension, or respiratory distress. Differential diagnoses such as pulmonary embolism, sepsis, anaphylaxis, and myocardial infarction must be excluded.[4]

Management of AFE focuses on supportive measures for rapid maternal cardiopulmonary stabilization.[4] The most important goal of therapy is to prevent hypoxia, cardiologic shock, and subsequent multiorgan failure. General supportive managements include supplemental oxygen, fluid resuscitation and transfusion to correct hypoxia, hypotension, and DIC, respectively.[2]

ECMO has been suggested as a new strategy in the treatment of AFE for rapid cardiopulmonary stabilization. There are a few case reports about the successful application of ECMO in patients with AFE.[9-12] In these cases, the goal was to recover from cardiogenic shock; thus, venoarterial ECMO was used. In the present case, AFE was suspected because of the sudden acute respiratory failure combined with DIC. However, other cardinal signs such as cardiogenic shock and altered mental status were not observed.[13] VV ECMO is mainly used for lung support, so we decided to provide VV ECMO to our patient.

DIC is present in $50 \%$ to $83 \%$ of patients with AFE. [3,6] Although the precise mechanism of the association between
DIC and AFE remains unclear, the current hypothesis is that the presence of tissue factor in amniotic fluid activates the extrinsic pathway, triggering clotting by activation of factor $\mathrm{X}$, and inducing the development of a consumptive coagulopathy.[14] A common complication of ECMO is bleeding because systemic anticoagulation with heparin is needed to avoid circuit thrombosis.[15] Sharma et al.[16] suggested that ECMO specialists should focus on the continuous adjustment of anticoagulation parameters, replacement of blood products, and bleeding control in patients with AFE. Although profound hemorrhage and bleeding were not found in our patient, nafamostat mesilate was used as an anticoagulant to reduce hemorrhagic complications.

In conclusion, AFE is a rare but potentially fatal complication of pregnancy, the postpartum period, and D\&C. AFE is unpredictable, so suspicion of the disease and prompt, aggressive resuscitation measures are important to improve clinical outcomes. Early application of ECMO should be considered for the treatment of patients with AFE unresponsive to medical therapy before massive organ damage.

\section{References}

1) Kanayama N, Tamura N: Amniotic fluid embolism: pathophysiology and new strategies for management. J Obstet Gynaecol Res 2014; 40: 1507-17.

2) Moore J, Baldisseri MR: Amniotic fluid embolism. Crit Care Med 2005; 33(10 Suppl): S279-85.

3) Clark SL, Hankins GD, Dudley DA, Dildy GA, Porter TF: Amniotic fluid embolism: analysis of the national registry. Am J Obstet Gynecol 1995; 172(4 Pt 1): 115867; discussion 1167-9.

4) Clark SL: Amniotic fluid embolism. Obstet Gynecol 2014; 123(2 Pt 1): 337-48.

5) Conde-Agudelo A, Romero R: Amniotic fluid embolism: an evidence-based review. Am J Obstet Gynecol 2009; 201: 445.e1-13.

6) Pantaleo G, Luigi N, Federica T, Paola S, Margherita N, Tahir M: Amniotic fluid embolism: review. Curr Pharm Biotechnol 2014; 14: 1163-7.

7) de Swiet M: Maternal mortality: confidential enquiries into maternal deaths in the United Kingdom. Am J Obstet Gynecol 2000; 182: 760-6. 
8) Yoneyama K, Sekiguchi A, Matsushima T, Kawase R, Nakai A, Asakura H, et al: Clinical characteristics of amniotic fluid embolism: an experience of 29 years. J Obstet Gynaecol Res 2014; 40: 1862-70.

9) Ho CH, Chen KB, Liu SK, Liu YF, Cheng HC, Wu RS: Early application of extracorporeal membrane oxygenation in a patient with amniotic fluid embolism. Acta Anaesthesiol Taiwan 2009; 47: 99-102.

10) Hsieh YY, Chang CC, Li PC, Tsai HD, Tsai CH: Successful application of extracorporeal membrane oxygenation and intra-aortic balloon counterpulsation as lifesaving therapy for a patient with amniotic fluid embolism. Am J Obstet Gynecol 2000; 183: 496-7.

11) Ecker JL, Solt K, Fitzsimons MG, MacGillivray TE: Case records of the Massachusetts General Hospital. Case 40-2012. A 43-year-old woman with cardiorespiratory arrest after a cesarean section. N Engl J Med 2012; 367: 2528-36.

12) Reyftmann L, Morau E, Dechaud H, Frapier JM, Hedon B: Extracorporeal membrane oxygenation therapy for circulatory arrest due to postpartum hemorrhage. Obstet Gynecol 2006; 107(2 Pt 2): 511-4.

13) Ito F, Akasaka J, Koike N, Uekuri C, Shigemitsu A, Kobayashi $\mathrm{H}$ : Incidence, diagnosis and pathophysiology of amniotic fluid embolism. J Obstet Gynaecol 2014; 34: 580-4.

14) Uszyński M, Zekanowska E, Uszyński W, Kuczyński J: Tissue factor (TF) and tissue factor pathway inhibitor (TFPI) in amniotic fluid and blood plasma: implications for the mechanism of amniotic fluid embolism. Eur J Obstet Gynecol Reprod Biol 2001; 95: 163-6.

15) Allen S, Holena D, McCunn M, Kohl B, Sarani B: A review of the fundamental principles and evidence base in the use of extracorporeal membrane oxygenation (ECMO) in critically ill adult patients. J Intensive Care Med 2011; 26: 13-26.

16) Sharma NS, Wille KM, Bellot SC, Diaz-Guzman E: Modern use of extracorporeal life support in pregnancy and postpartum. ASAIO J 2015; 61: 110-4. 